\title{
Fatores associados ao controle glicêmico em amostra de indivíduos com diabetes mellitus do Estudo Longitudinal de Saúde do Adulto, Brasil, 2008 a 2010*
}

doi: $10.5123 /$ S1679-49742020000300017

\author{
Factors associated with glycemic control in a sample of individuals with Diabetes Mellitus \\ taken from the Longitudinal Study of Adult Health, Brazil, 2008-2010
}

\section{Factores asociados con el control glucémico en una muestra de individuos con diabetes Mellitus del Estudio Longitudinal de la Salud de Adultos, Brasil, 2008-2010}

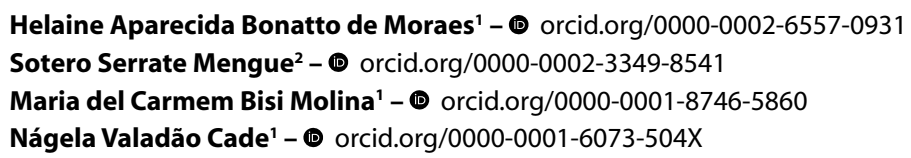

\section{Resumo}

Objetivo: investigar os fatores associados ao controle glicêmico em indivíduos com diabetes mellitus (DM). Métodos: estudo seccional, com participantes do Estudo Longitudinal de Saúde do Adulto com DM autorreferido; utilizou-se regressão logística binomial. Resultados: foram incluídos 1.242 indivíduos; 54,2\% apresentaram hemoglobina glicada $\geq 6,5 \%$, evidenciando controle glicêmico inadequado; mostraram-se fatores associados ao controle glicêmico inadequado o sexo masculino $(\mathrm{OR}=1,39$ - $\left.\mathrm{IC}_{95 \%} 1,05 ; 1,85\right)$, raça/cor da pele preta $\left(\mathrm{OR}=1,74-\mathrm{IC}_{95 \%} 1,22 ; 2,48\right)$ ou parda $\left(\mathrm{OR}=1,57\right.$ - $\left.\mathrm{IC}_{95 \%} 1,14 ; 2,16\right)$, nível médio de ocupação $\left(\mathrm{OR}=1,63-\mathrm{IC}_{95 \%} 1,02 ; 2,58\right)$, não ter plano de saúde $\left(\mathrm{OR}=1,47-\mathrm{IC}_{95 \%} 1,09 ; 1,96\right)$, uso de insulina $(0 \mathrm{R}=7,34$ - $\left.\mathrm{IC}_{95 \%} 3,56 ; 15,15\right)$, relação cintura-quadril alterada $\left(\mathrm{OR}=1,87-\mathrm{IC}_{95 \%} 1,19 ; 2,93\right)$, tabagismo $\left(0 \mathrm{R}=1,73 \mathrm{IC}_{95 \%} 1,09 ; 2,74\right) \mathrm{e}$ autoavaliação da saúde ruim ou muito ruim $\left(0 \mathrm{R}=2,37-\mathrm{IC}_{95 \%} 1,17 ; 4,83\right)$. Conclusão: os resultados reforçam o contexto multicausal no controle glicêmico, que foi associado a fatores sociodemográficos, estilos de vida e condições de saúde.

Palavras-chave: Diabetes Mellitus; Hemoglobina A Glicada; Complicações do Diabetes; Hiperglicemia.

*Artigo derivado de dissertação de mestrado 'A não associação entre adesão medicamentosa e o controle glicêmico em participantes diabéticos do ELSA-Brasil', defendida por Helaine Aparecida Bonatto de Moraes junto ao Programa de Pós-Graduação em Saúde Coletiva da Universidade Federal do Espírito Santo (UFES) em 2017.

Endereço para correspondência:

Helaine Aparecida Bonatto de Moraes - Rua João Benedito, n² 21, Colatina, Espírito Santo, Brasil. CEP: 29707-082

E-mail:habonatto@yahoo.com.br 


\section{Introdução}

0 diabetes mellitus (DM) representa, atualmente, uma epidemia mundial, um grande desafio para os sistemas nacionais de saúde. Fatores como a urbanização e industrialização, 0 aumento global da expectativa de vida e estilo de vida caracterizado por inatividade física e hábitos alimentares que predispõem ao acúmulo de gordura corporal têm contribuído para 0 avanço da epidemia do DM em todo o mundo. ${ }^{1}$

0 Brasil é o quarto país com mais casos da doença em adultos no mundo (14,3 milhões de indivíduos); apenas no ano de 2015, ocorreram 130.700 mortes causadas pelo DM. ${ }^{2}$ Inquérito domiciliar sobre a ocorrência do DM, realizado no país em 2013, revelou que a prevalência da doença autorreferida foi de $6,2 \%$, com maior proporção entre as mulheres e os moradores de áreas urbanas. ${ }^{3}$

\section{O Brasil é o quarto país com mais casos da doença em adultos no mundo (14,3 milhões de indivíduos); apenas no ano de 2015, ocorreram 130.700 mortes causadas pelo DM.}

0 DM é compreendido como um grupo heterogêneo de distúrbios metabólicos cuja característica marcante é a hiperglicemia. No DM tipo 1, há uma ausência de produção de insulina pelo organismo e seu tratamento, obrigatoriamente, necessita de insulina exógena; 0 DM tipo 2, responsável por mais de $90 \%$ dos casos, está relacionado a defeitos na ação, secreção da insulina e regulação da produção hepática de glicose, e seu tratamento implica a tomada de uma série de medidas para obtenção do controle dietético que incluem o combate à obesidade, promoção de atividade física regular e uso de medicamentos antidiabéticos orais, isolados ou combinados. ${ }^{1}$

Para avaliação do controle glicêmico, a hemoglobina A glicosilada (hemoglobina A1c) se destaca como exame padrão. ${ }^{1}$ Há amplas evidências de que 0 bom controle da glicemia e dos demais fatores de risco, por exemplo, a obesidade, a inatividade física e uma dieta hipercalórica, previnem tanto as complicações agudas quanto as crônicas da doença. Estudo avaliativo com indivíduos portadores de DM descompensados identificou que, no início do acompanhamento, nenhum paciente estava com todos os parâmetros (controle glicêmico, pressórico e lipídico) dentro dos limites das metas preconizadas. ${ }^{4}$ Autores chamam a atenção sobre a importância de mudanças nos hábitos de vida, para 0 controle do peso, dieta e atividade física. ${ }^{5}$ Nesse sentido, compreender os fatores determinantes para 0 controle do DM tem-se revelado fundamental: quanto mais precocemente a intervenção é realizada, melhor o curso clínico da doença e menor a probabilidade do surgimento de suas complicações. ${ }^{1,5}$

Este estudo teve por objetivo investigar os fatores associados ao controle glicêmico de indivíduos com DM, participantes do Estudo Longitudinal de Saúde do Adulto (ELSA-Brasil).

\section{Métodos}

Trata-se de um estudo seccional com dados da linha de base do Estudo Longitudinal de Saúde do Adulto (ELSA-Brasil), cujas informações foram coletadas entre agosto de 2008 e dezembro de $2010 .^{6}$

A coorte do ELSA-Brasil foi composta por 15.105 servidores públicos ativos ou aposentados, com idade entre 35 e 74 anos, de seis instituições públicas de ensino superior das seguintes capitais dos estados brasileiros: São Paulo (São Paulo), Belo Horizonte (Minas Gerais), Salvador (Bahia), Porto Alegre (Rio Grande do Sul), Rio de Janeiro (Rio de Janeiro) e Vitória (Espírito Santo). ${ }^{6}$

Para a coleta de dados da linha de base do ELSA-Brasil, equipes capacitadas e certificadas em nível central conduziram a aplicação de entrevistas padronizadas, avaliações clínicas e coleta de material para a realização de exames bioquímicos. A cada três anos, os participantes são contatados para a realização de novos procedimentos, visando ao acompanhamento da saúde e monitoramento dos desfechos individuais. ${ }^{7}$

0 estudo incluiu todos os indivíduos que participaram da linha de base do ELSA-Brasil com DM autorreferido e em uso de antidiabético(s) oral(is) ou insulina. Foram excluídos da amostra os que não apresentaram resultados para todos os parâmetros bioquímicos estudados e para a variável 'adesão medicamentosa'.

A variável 'desfecho' deste estudo foi a hemoglobina glicada (HbA1c). A Sociedade Brasileira de Diabetes, em sua diretriz mais recente, define, como critério diagnóstico para DM estabelecido, valores de HbA1c maiores e/ou iguais a 6,5\%. ${ }^{1}$ Nesta investigação, para avaliar o controle glicêmico, foram utilizados os dados laboratoriais da hemoglobina glicada (HbA1c), cujo 
resultado foi considerado adequado quando apresentou valor menor que $6,5 \%{ }^{8}$

As variáveis de exposição foram das seguintes naturezas:

a) socioeconômica e demográfica

- idade (em anos: 35 a 44; 45 a 54; 55 a 64; 65 ou mais)

- sexo (masculino; feminino);

- raça/cor da pele (branca; preta; parda; amarela/ indígena);

- estado civil (casado/vive com alguém; divorciado ou separado; solteiro);

- escolaridade (ensino fundamental incompleto; ensino fundamental completo; ensino médio completo; ensino superior); $\mathrm{e}$

- renda per capita (em salários mínimos: menos de 4; de 4 a menos de 8; de 8 a menos de 12; de 12 a menos de $16 ; 16$ ou mais);

b) hábitos de vida

- atividade física (ativo; não ativo);

- consumo de álcool (nunca usou; ex-usuário; usuário); e

- tabagismo (nunca fumou; fumante; ex-fumante);

c) alimentar

- calorias totais diárias da dieta $(<2.000 ; 2.000<3.000$; $3.000<4.000 ; \geq 4.000)$;

d) nutricional, mensurada com base

- índice de massa corporal (IMC) - baixo peso (IMC $\left.<18,5 \mathrm{~kg} / \mathrm{m}^{2}\right) /$ eutrofia $\left(\right.$ IMC $\left.<25 \mathrm{~kg} / \mathrm{m}^{2}\right)$; sobrepeso $\left(\right.$ IMC $\left.<30 \mathrm{~kg} / \mathrm{m}^{2}\right) ;$ obesidade (IMC $\geq 30 \mathrm{~kg} / \mathrm{m}^{2}$ ) -;

- relação cintura-quadril (ideal; alterada); e

- circunferência abdominal (ideal; alterada);

e) psicossocial

- atividade religiosa (não; sim); e

- autoavaliação de saúde (muito boa; boa; regular; ruim/muito ruim);

f) características gerais e de saúde

- categoria funcional (em nível de escolaridade admitido para a função: apoio; médio [cargo técnico]; superior/docente);

- plano de saúde privado (não; sim; sem informação);

g) medicação para DM (antidiabético oral; insulina; ambos); $\mathrm{e}$

h) adesão ao tratamento medicamentoso (baixa/media adesão; alta adesão).

Sobre a variável 'renda per capita', o valor do salário mínimo vigente em 2008 era de R \$ 415,00 (US\$257,44).
A variável 'autoavaliação de saúde' foi aferida pela pergunta "De um modo geral, em comparação a pessoas da sua idade, como o(a) senhor (a) considera o seu estado de saúde?", cujas opções de resposta foram categorizadas em estratos: muito bom; bom; regular; ruim; muito ruim; sem informação (NS [não sabe]/ $\mathrm{NQR}$ [não quis responder]).

Na variável 'consumo de álcool', a categoria 'usuário' foi registrada mediante a pergunta "Atualmente consome bebida alcoólica?" e as opções de resposta 'não' ou 'sim'; a categoria 'ex-usuário', pela pergunta "Já consumiu bebidas alcoólicas?" e as respostas 'não' ou 'sim'; e a categorização 'nunca usou', atribuída aos indivíduos que informaram jamais ter feito uso de álcool.

Para a definição da variável 'calorias da dieta', 0 valor energético total foi investigado com a aplicação do Questionário de Frequência Alimentar (QFA ELSA-Brasil), previamente validado, contendo 114 itens alimentares. A seguir, foram estimadas a composição nutricional e 0 valor energético dos alimentos consumidos e, por fim, realizada a categorização numérica do valor energético total da dieta, em calorias (cal), para cada indivíduo.

A variável 'categoria funcional' foi definida segundo o nível de escolaridade exigido para a ocupação do cargo público: apoio; técnico; ou superior/docente. A categoria 'apoio' representa o conjunto de ocupações para as quais se exige 0 ensino fundamental completo.

A variável 'adesão medicamentosa' foi avaliada com a aplicação da Morisky Medication Adherence Scale de quatro itens (MMAS-4),${ }^{10}$ segundo a qual, para cada resposta positiva atribui-se 1 (um) ponto, e para cada resposta negativa a pontuação 0 (zero). A adesão foi classificada da seguinte forma: alta, quando as 4 respostas eram negativas; média, quando 3 ou 2 respostas eram negativas; e baixa quando apenas uma resposta era negativa, ou quando todas as respostas eram positivas. Considerou-se aderente aos medicamentos 0 indivíduo classificado pela MMAS-4 com alta adesão, e não aderente aquele classificado com média ou baixa adesão. ${ }^{11}$ Apesar de 0 MMAS-4 ter baixa sensibilidade $(43,6 \%)$ e razoável especificidade (81\%), ${ }^{12}$ essa escala continua a ser utilizada em muitos estudos. ${ }^{10-12}$

A circunferência da cintura (CC) foi categorizada como ideal nos indivíduos homens que apresentaram valor menor do que $90 \mathrm{~cm}$, e nas mulheres quando esse valor foi menor do que $80 \mathrm{~cm}$, de acordo com a recomendação da International Diabetes Federation. ${ }^{13}$ Já a relação cintura-quadril (RCQ), avaliada segundo 
os critérios da Associação Brasileira para Estudo da Obesidade e da Síndrome Metabólica, foi categorizada como ideal quando se identificaram valores menores do que 0,90 para homens e 0,85 para mulheres. ${ }^{14}$

A determinação da atividade física pautou-se nas respostas ao International Physical Activity Questionnaire (IPAQ), em sua versão longa, sendo considerados como suficientemente ativos os indivíduos que relataram no mínimo 150 minutos de atividade física moderada por semana. ${ }^{15}$ As variáveis relacionadas ao tratamento medicamentoso consistiram da descrição do perfil de utilização dos medicamentos, distribuído em três categorias: hipoglicemiante oral; uso de insulina; ou ambos.

$\mathrm{Na}$ análise de dados, a comparação entre variáveis categóricas foi feita pelo teste do qui-quadrado de Pearson. A análise multivariável foi realizada mediante regressão logística binomial, tendo como categoria de referência a hemoglobina glicada $<6,5 \%$, e incluiu todas as variáveis associadas ao resultado com p-valor $<0,100$ na análise bruta. As análises estatísticas utilizaram-se dos aplicativos SPSS (Statistical Package for the Social Sciences, ou Pacote Estatístico para Ciências Sociais), em sua versão 22, e BioEstat 5.3; o nível de significância adotado foi de $5 \%$.

O Projeto ELSA-Brasil foi aprovado pelo Comitê Nacional de Ética em Pesquisa (CONEP)/Conselho Nacional de Saúde (CNS), em 4 de agosto de 2006, sob o $n^{0} 13.065$, e pelos Comitês de Ética em Pesquisa (CEP) das seis instituições integrantes do consórcio. 0 estudo foi submetido e aprovado pelo Comitê Científico do ELSA-Brasil.

\section{Resultados}

Foram incluídos 1.242 indivíduos no estudo. A prevalência de DM na população estudada foi de 8,2\%. Houve predomínio de homens (53,1\%); 41,4\% dos participantes se encontravam na faixa etária de 55 a 64 anos, $43,9 \%$ eram de raça/cor da pele preta, 78,4\% tinham ensino médio e superior, $61,2 \%$ referiam renda per capita menor do que quatro salários mínimos $25,3 \%$ recebiam valores entre quatro e menos de oito salários mínimos (Tabela 1).

Houve predomínio de pessoas fisicamente inativas $(96,6 \%)$, que nunca fumaram $(50,6 \%)$ e que consumiam bebida alcoólica (56,2\%). Dos 697 indivíduos autodeclarados usuários de álcool, 10,2\% foram classificados como bebedores excessivos (Tabela 1).
A maioria $(60,2 \%)$ tinha baixa/média adesão à medicação e 54,2\% dos indivíduos com DM apresentavam controle glicêmico inadequado (Tabela 1).

Quanto às categorias de fármacos usados pelos indivíduos com DM, 86,5\% utilizavam apenas 0 antidiabético oral, 7,8\% usavam antidiabéticos orais e insulina concomitante, e 5,7\% usavam somente insulina como forma de tratamento (Tabela 1). De acordo com a classe farmacológica dos antidiabéticos orais e respectivos princípios ativos, dos 1.174 indivíduos com DM em uso de antidiabéticos orais, a maioria fazia uso isolado de biguanida $(57,2 \%)$, seguida dos que se utilizavam de biguanida e sulfunilureia associadas (24,2\%); somente $8,9 \%$ usavam sulfunilureia isolada; outras associações medicamentosas perfizeram 9,5\%. Do total da amostra, $0,2 \%$ não souberam informar sobre os fármacos utilizados por eles. Não estava disponível no banco de dados do Projeto ELSA-Brasil alguma informação sobre o número de medicamentos utilizados por cada indivíduo portador de diabetes.

Na Tabela 2, observa-se que sobrepeso e obesidade, somados, representaram $82,8 \%$ da amostra, circunferência da cintura e RCQ alterados, mais de $90 \%$ cada um, e quase $40 \%$ acusaram obesidade abdominal. Autoavaliaram a saúde como boa $45,8 \%$ da amostra, e 63,4\% tinham plano de saúde.

$\mathrm{Na}$ análise bruta (Tabelas 3 e 4), o controle glicêmico adequado (HbAlc $<6,5 \%$ ) apresentou maior proporção em pessoas do sexo feminino, de raça/cor da pele branca, com nível de escolaridade superior, que utilizavam apenas antidiabético oral, com atividade religiosa e plano de saúde, que nunca fumaram, com autoavaliaçäo de saúde boa e consumo de dieta de 2.000 a 3.000 calorias diárias. Pior adesão medicamentosa e pior renda per capita foram observadas naqueles com pior controle glicêmico (HbA1c $\geq 6,5 \%$ ).

$\mathrm{Na}$ análise multivariável (Tabela 5), foram associadas ao controle glicêmico inadequado as seguintes variáveis: sexo masculino $\left(\mathrm{OR}=1,39-\mathrm{IC}_{95 \%} 1,05 ; 1,85\right)$; raça/cor da pele preta $\left(\mathrm{OR}=1,74-\mathrm{IC}_{95 \%} 1,22 ; 2,48\right)$ ou parda $\left(\mathrm{OR}=1,57-\mathrm{IC}_{95 \%} 1,14 ; 2,16\right)$; uso de insulina isoladamente $\left(\mathrm{OR}=7,34-\mathrm{IC}_{95 \%} 3,56 ; 15,15\right)$ ou associada a antidiabético oral $\left(\mathrm{OR}=7,58-\mathrm{IC}_{95 \%} 3,96 ; 14,52\right)$; relação cintura-quadril (RCQ) alterada $(\mathrm{OR}=1,87$ - IC $\left.{ }_{95 \%} 1,19 ; 2,93\right)$; ocupação compatível com nível médio (cargo técnico) de instrução $(\mathrm{OR}=1,63-$ $\left.\mathrm{IC}_{95 \%} 1,02 ; 2,58\right)$, comparada à de quem possuía nível superior; autoavaliação da saúde ruim ou muito ruim 
Tabela 1 - Caracterização das variáveis socioeconômicas, demográficas, renda e hábitos de vida e de saúde dos indivíduos com diabetes mellitus participantes da linha de base do Estudo Longitudinal de Saúde do Adulto, Brasil, 2008-2010

\begin{tabular}{|c|c|c|c|}
\hline Variável & Categorização & $\mathrm{n}$ & $\%$ \\
\hline \multirow{2}{*}{ Sexo } & Masculino & 659 & 53,1 \\
\hline & Feminino & 583 & 46,9 \\
\hline \multirow{4}{*}{ Faixa etária (em anos) } & $35-44$ & 80 & 6,4 \\
\hline & $45-54$ & 354 & 28,5 \\
\hline & $55-64$ & 514 & 41,4 \\
\hline & $\geq 65$ & 294 & 23,7 \\
\hline \multirow{5}{*}{ Raça/cor da pele } & Preta & 545 & 43,9 \\
\hline & Parda & 273 & 22,0 \\
\hline & Branca & 343 & 27,6 \\
\hline & Amarela/indígena & 61 & 4,9 \\
\hline & Sem informação & 20 & 1,6 \\
\hline \multirow{4}{*}{ Escolaridade } & $1^{\circ}$ grau incompleto & 143 & 11,5 \\
\hline & $1^{\circ}$ grau completo & 125 & 10,1 \\
\hline & $2^{\circ}$ grau completo & 464 & 37,3 \\
\hline & Superior & 510 & 41,1 \\
\hline \multirow{4}{*}{ Estado civil } & Casado ou vive com alguém & 808 & 65,1 \\
\hline & Divorciado, separado ou viúvo & 308 & 24,8 \\
\hline & Solteiro & 125 & 10,0 \\
\hline & Sem informação & 1 & 0,1 \\
\hline \multirow{6}{*}{$\begin{array}{l}\text { Renda per capita } \\
\text { (em salários mínimos) }\end{array}$} & $<4$ & 760 & 61,2 \\
\hline & $4 a<8$ & 315 & 25,3 \\
\hline & $8 a<12$ & 120 & 9,7 \\
\hline & $12 a<16$ & 11 & 0,9 \\
\hline & $\geq 16$ & 31 & 2,5 \\
\hline & Sem informação & 5 & 0,4 \\
\hline \multirow{2}{*}{ Atividade física } & Ativo & 42 & 3,4 \\
\hline & Não ativo & 1.200 & 96,6 \\
\hline \multirow{4}{*}{ Hábito de fumar } & Nunca fumou & 629 & 50,6 \\
\hline & Ex-fumante & 480 & 38,6 \\
\hline & Fumante & 132 & 10,7 \\
\hline & Sem informação & 1 & 0,1 \\
\hline \multirow{3}{*}{ Consumo de álcool } & Nunca usou & 176 & 14,1 \\
\hline & Ex-usuário & 369 & 29,7 \\
\hline & Usuário & 697 & 56,2 \\
\hline \multirow{2}{*}{ Adesão medicamentosa } & Baixa/média adesão & 748 & 60,2 \\
\hline & Alta adesão & 494 & 39,8 \\
\hline \multirow{3}{*}{ Tipos de medicamentos } & Antidiabético oral & 1.074 & 86,5 \\
\hline & Insulina & 71 & 5,7 \\
\hline & Ambos & 97 & 7,8 \\
\hline \multirow{2}{*}{ Controle glicêmico } & Hemoglobina glicada $\geq 6,5 \%$ & 673 & 54,2 \\
\hline & Hemoglobina glicada $<6,5 \%$ & 569 & 45,8 \\
\hline Total & & 1.242 & 100,0 \\
\hline
\end{tabular}

$\left(\mathrm{OR}=2,37-\mathrm{IC}_{95 \%} 1,17 ; 4,83\right) ;$ não ter plano privado de saúde ( $\left.0 \mathrm{R}=1,47-\mathrm{IC}_{95 \%} 1,09 ; 1,96\right)$; e ser tabagista $\left(\mathrm{OR}=1,73-\mathrm{IC}_{95 \%}, 1,09 ; 2,74\right)$.

\section{Discussão}

A prevalência do diabetes mellitus na população estudada foi de $8,2 \%$. 0 estudo evidenciou que os fatores associados a maior chance do controle glicêmico inadequado incluíram variáveis socioeconômicas e demográficas (sexo masculino; raça/cor da pele preta ou parda), estilo de vida (tabagismo; relação cintura-quadril aumentada), condição de saúde do indivíduo (uso de insulina; autoavaliação da saúde ruim), além da categoria funcional compatível com ensino médio (cargo técnico) e não possuir plano de saúde. 
Tabela 2 - Caracterização das variáveis nutricionais, alimentares, psicossociais e funcionais dos indivíduos com diabetes mellitus participantes da linha de base do Estudo Longitudinal de Saúde do Adulto, Brasil, 2008-2010

\begin{tabular}{|c|c|c|c|}
\hline Variável & Categorização & $\mathrm{n}$ & $\%$ \\
\hline \multirow{3}{*}{ Índice de massa corporal ( IMC) } & Baixo peso/eutrofia & 214 & 17,2 \\
\hline & Sobrepeso & 518 & 41,7 \\
\hline & Obesidade & 510 & 41,1 \\
\hline \multirow{3}{*}{ Circunferência da cintura (CC) } & Ideal & 122 & 9,8 \\
\hline & Alterada & 1.119 & 90,1 \\
\hline & Sem informação & 1 & 0,1 \\
\hline \multirow{3}{*}{ Relação cintura-quadril (RCQ) } & Ideal & 117 & 9,4 \\
\hline & Alterada & 1.124 & 90,5 \\
\hline & Sem informação & 1 & 0,1 \\
\hline \multirow{3}{*}{ Obesidade abdominal } & Não & 315 & 25,4 \\
\hline & Sim & 484 & 39,0 \\
\hline & Sem informação & 443 & 35,6 \\
\hline \multirow{5}{*}{ Calorias totais diárias da dieta } & $<2.000$ & 265 & 21,3 \\
\hline & $2.000<3.000$ & 567 & 45,7 \\
\hline & $3.000<4.000$ & 255 & 20,5 \\
\hline & $\geq 4.000$ & 154 & 12,4 \\
\hline & Sem informação & 1 & 0,1 \\
\hline \multirow{5}{*}{ Autoavaliação de saúde (AAS) } & Muito boa & 108 & 8,7 \\
\hline & Boa & 569 & 45,8 \\
\hline & Regular & 483 & 38,9 \\
\hline & Ruim/muito ruim & 80 & 6,4 \\
\hline & Sem informação & 2 & 0,2 \\
\hline \multirow{3}{*}{ Plano privado de saúde } & Não & 454 & 36,5 \\
\hline & Sim & 787 & 63,4 \\
\hline & Sem informação & 1 & 0,1 \\
\hline \multirow{3}{*}{ Histórico ocupacional } & Ativo & 754 & 60,7 \\
\hline & Aposentado & 487 & 39,2 \\
\hline & Sem informação & 1 & 0,1 \\
\hline \multirow{3}{*}{ Categoria funcional } & Apoio & 404 & 32,5 \\
\hline & Técnico & 452 & 36,4 \\
\hline & Superior/docente & 386 & 31,1 \\
\hline \multirow{2}{*}{ Atividade religiosa } & Não & 354 & 28,5 \\
\hline & Sim & 888 & 71,5 \\
\hline Total & & 1.242 & 100,0 \\
\hline
\end{tabular}

Entre as variáveis socioeconômicas e demográficas, o sexo masculino foi associado a piores resultados de níveis glicêmicos. Outro estudo de âmbito nacional, com portadores de DM autorreferido, também indicou diferença entre os sexos, com maior prevalência no sexo feminino $\left(7,0 \%\right.$ - $\left.\mathrm{IC}_{95 \%} 6,5 ; 7,5\right)$, fato este creditado à maior procura das mulheres pelo serviço de saúde, especialmente nos períodos de gestação, além da maior consciência feminina da importância do cuidado com a própria saúde. ${ }^{3}$

Não obstante a literatura apontar mais casos da doença em mulheres, neste estudo em particular, os homens apresentaram maior descontrole glicêmico. Essa relação entre os sexos pode-se atribuir a uma série de fatores comportamentais e culturais de saúde dos homens, para muitos dos quais a doença pode ser considerada um sinal de fragilidade, levando-os a se esquivarem das consultas e do cuidado com a saúde. ${ }^{16}$ Há que se considerar, também, o medo do público masculino da descoberta de uma doença grave, mais uma razão para explicar a ausência dos homens nos serviços de saúde, dificultando o estabelecimento de hábitos de prevenção entre eles. ${ }^{17}$

Neste estudo, 0 indivíduo ser de raça/cor da pele preta ou parda aumentou a chance de pior controle glicêmico. Da mesma forma, outra pesquisa identificou diferenças étnicas no surgimento do DM, com valores duas vezes maiores da doença em pessoas de etnia negra. ${ }^{18}$ Apesar 
Tabela 3 - Variáveis socioeconômicas, demográficas, tipos de medicamentos e adesão dos indivíduos com diabetes mellitus, segundo o controle glicêmico, participantes da Linha de base do Estudo Longitudinal de Saúde do Adulto, Brasil, 2008-2010

\begin{tabular}{|c|c|c|c|c|}
\hline \multirow[t]{2}{*}{ Variável } & \multirow[t]{2}{*}{ Categorização } & \multicolumn{2}{|c|}{$\begin{array}{c}\text { Controle glicêmico inadequado } \\
(\mathrm{HbA1c} \geq 6,5 \%)\end{array}$} & \multirow[t]{2}{*}{ p-valor } \\
\hline & & $n$ & $\%$ & \\
\hline \multirow{2}{*}{ Sexo } & Masculino & 377 & 57,2 & \multirow{2}{*}{0,023} \\
\hline & Feminino & 296 & 50,8 & \\
\hline \multirow{4}{*}{ Faixa etária (em anos) } & $35-44$ & 46 & 57,5 & \multirow{4}{*}{0,080} \\
\hline & $45-54$ & 210 & 59,3 & \\
\hline & $55-64$ & 270 & 52,5 & \\
\hline & $\geq 65$ & 147 & 50,0 & \\
\hline \multirow{4}{*}{ Raça/cor da pele } & Preta & 244 & 44,8 & \multirow{4}{*}{$<0,001$} \\
\hline & Parda & 177 & 64,8 & \\
\hline & Branca & 209 & 60,9 & \\
\hline & Amarela/indígena & 35 & 57,4 & \\
\hline \multirow{4}{*}{ Escolaridade } & $1^{\circ}$ grau incompleto & 101 & 70,6 & \multirow{4}{*}{$<0,001$} \\
\hline & $1^{\circ} \mathrm{grau}$ completo & 89 & 71,2 & \\
\hline & $2^{\circ}$ grau completo & 274 & 59,1 & \\
\hline & Superior & 209 & 40,9 & \\
\hline \multirow{5}{*}{$\begin{array}{l}\text { Renda per capita (em salários } \\
\text { mínimos) }\end{array}$} & $<4$ & 470 & 61,8 & \multirow{5}{*}{$<0,001$} \\
\hline & $4 a<8$ & 138 & 43,8 & \\
\hline & $8 a<12$ & 48 & 40,0 & \\
\hline & $12 a<16$ & 6 & 54,5 & \\
\hline & $\geq 16$ & 9 & 29,0 & \\
\hline \multirow{3}{*}{ Estado civil } & Casado/vive com alguém & 437 & 54,0 & \multirow{3}{*}{0,587} \\
\hline & Divorciado ou separado & 172 & 55,8 & \\
\hline & Solteiro & 63 & 50,4 & \\
\hline \multirow{3}{*}{ Tipo de medicamentos } & Antidiabético oral & 528 & 49,2 & \multirow{3}{*}{$<0,001$} \\
\hline & Insulina & 61 & 85,9 & \\
\hline & Ambos & 85 & 87,6 & \\
\hline \multirow{2}{*}{ Adesão medicamentosa } & Baixa/média & 426 & 56,9 & \multirow{2}{*}{0,016} \\
\hline & Alta & 247 & 50,0 & \\
\hline
\end{tabular}

a) p-valor: calculado pelo teste do qui-quadrado de Pearson.

de estes estudos apresentarem as mesmas constatações frente a raça/cor da pele negra/parda, relativamente ao surgimento e ao controle da doença, essas disparidades não se veem tão bem estabelecidas: para alguns autores, elas se devem à dificuldade de superar iniquidades, como as diferenças na educação formal, na comunicação e no acesso aos serviços de saúde, reiterando a existência de disparidade étnica e social na evolução do DM, além da necessidade de uma discussão sobre a identificação das barreiras possivelmente relacionadas a um pior controle da doença por indivíduos de raça/ cor da pele preta ou parda. ${ }^{19}$

Indivíduos cuja categoria funcional era compatível com nível de escolaridade médio (cargo técnico) tiveram mais chance de apresentar controle glicêmico inadequado, quando comparados àqueles com nível superior. Esta associação entre menos anos de estudo e mais chances de ter DM também esteve presente em outros estudos. ${ }^{20,21}$ Após comparação entre as categorias funcionais estudadas, indivíduos de nível superior constituem, em sua maioria, docentes e pesquisadores, os quais, pela natureza das instituições envolvidas, possuem vantagens em relação às demais categorias funcionais (técnico e apoio), como também maior flexibilidade de horários, férias superiores a 30 dias/ano e carga de trabalho adaptável a outros ambientes, de forma a se pensar no fator contribuite dessas condições para as diferenças observadas no processo de cuidado com a saúde e no controle de doenças crônicas, caso do DM. A verificação do descontrole glicêmico em indivíduos com menor escolaridade vem reforçar a importância da questão educacional no processo de conhecimento da doença e nas atitudes tomadas frente a uma situação de saúde. Identificar como as pessoas vivem, trabalham e 
Tabela 4 - Características nutricionais, ocupacionais, psicossociais, hábitos de vida e dieta dos indivíduos com diabetes mellitus, segundo o controle glicêmico, participantes da linha de base do Estudo Longitudinal de Saúde do Adulto, Brasil, 2008-2010

\begin{tabular}{|c|c|c|c|c|}
\hline \multirow[t]{2}{*}{ Variável } & \multirow[t]{2}{*}{ Categorização } & \multicolumn{2}{|c|}{$\begin{array}{c}\text { Controle glicêmico inadequado } \\
(\mathrm{HbA1} \geq \geq 6,5 \%)\end{array}$} & \multirow[t]{2}{*}{$\mathrm{p}$-valor ${ }^{\mathrm{a}}$} \\
\hline & & $n$ & $\%$ & \\
\hline \multirow{3}{*}{ Índice de massa corporal (IMC) } & Baixo peso/eutrofia & 117 & 54,7 & \multirow{3}{*}{0,661} \\
\hline & Sobrepeso & 273 & 52,7 & \\
\hline & Obesidade & 283 & 55,5 & \\
\hline \multirow{2}{*}{ Circunferência da cintura (CC) } & Ideal & 65 & 53,3 & \multirow[t]{2}{*}{0,839} \\
\hline & Alterada & 607 & 54,2 & \\
\hline \multirow{2}{*}{ Relação cintura-quadril (RCQ) } & Ideal & 50 & 42,7 & \multirow[t]{2}{*}{0,009} \\
\hline & Alterada & 622 & 55,3 & \\
\hline \multirow{2}{*}{ Atividade religiosa } & Não & 176 & 49,7 & \multirow{2}{*}{0,046} \\
\hline & Sim & 497 & 55,9 & \\
\hline \multirow{3}{*}{ Categoria funcional } & Apoio & 273 & 67,6 & \multirow{3}{*}{$<0,001$} \\
\hline & Técnico & 260 & 57,5 & \\
\hline & Nível superior/docente & 140 & 36,3 & \\
\hline \multirow{2}{*}{ Plano privado de saúde } & Não & 300 & 66,1 & \multirow{2}{*}{$<0,001$} \\
\hline & Sim & 373 & 47,4 & \\
\hline \multirow{2}{*}{ Histórico ocupacional } & Ativo & 417 & 55,3 & \multirow{2}{*}{0,310} \\
\hline & Aposentado & 255 & 52,4 & \\
\hline \multirow{4}{*}{ Autoavaliação de saúde (AAS) } & Muito boa & 44 & 40,7 & \multirow{4}{*}{$<0,001$} \\
\hline & Boa & 278 & 48,8 & \\
\hline & Regular & 292 & 60,4 & \\
\hline & Ruim/muito ruim & 58 & 72,5 & \\
\hline \multirow{2}{*}{ Atividade física } & Ativo & 25 & 59,5 & \multirow{2}{*}{0,480} \\
\hline & Não ativo & 648 & 54,0 & \\
\hline \multirow{3}{*}{ Hábito de fumar } & Nunca fumou & 320 & 50,9 & \multirow{3}{*}{0,001} \\
\hline & Ex-fumante & 262 & 54,6 & \\
\hline & Fumante & 90 & 68,2 & \\
\hline \multirow{3}{*}{ Consumo de álcool } & Nunca usou & 102 & 57,9 & \multirow{3}{*}{0,039} \\
\hline & Ex-usuário & 214 & 58,0 & \\
\hline & Usuário & 355 & 50,9 & \\
\hline \multirow{4}{*}{ Calorias totais diárias da dieta } & $<2.000$ & 138 & 52,1 & \multirow{4}{*}{$<0,001$} \\
\hline & $2.000<3.000$ & 281 & 49,5 & \\
\hline & $3.000<4.000$ & 148 & 58,0 & \\
\hline & $\geq 4.000$ & 105 & 68,2 & \\
\hline
\end{tabular}

a) p-valor:calculado pelo teste do qui-quadrado de Pearson.

envelhecem demonstra a importância dos determinantes sociais, tanto no processo de adoecimento como no cuidado com a própria saúde..$^{22}$

Paralelamente a essas considerações, é indiscutível a importância da renda entre diferentes categorias funcionais. Profissionais de nível superior contam com remunerações mais atraentes, progressões mais vantajosas ao longo da carreira e, consequentemente, maior acesso aos serviços de saúde, inclusive com a aquisição de planos privados e utilização de medicamentos de primeira linha, de menos efeitos colaterais. Assim são entendidas essas questões enquanto determinantes sociais da saúde..$^{22}$
0 resultado deste trabalho mostrou que não dispor de plano privado de saúde também esteve associado a um controle glicêmico inadequado. Estudo que comparou o uso de serviços de saúde de diabéticos cobertos por planos privados com os usuários desses serviços pela Saúde Pública observou que o acesso (definido pela ausência de dificuldades para conseguir consultas médicas) foi mais alto entre os afiliados de um plano privado, contra usuários do serviços públicos cobertos pela Estratégia Saúde da Família (ESF) e unidade básica de saúde tradicional. ${ }^{23}$ Tal fato sugere a existência de importantes diferenças entre 0 acesso dos que possuem planos privados de saúde e os dependentes do serviço 
Tabela 5 - Fatores associados ao controle glicêmico em indivíduos com diabetes mellitus participantes da linha de base do Estudo Longitudinal de Saúde do Adulto, Brasil, 2008-2010

\begin{tabular}{|c|c|c|c|}
\hline \multirow{2}{*}{ Variável } & \multirow{2}{*}{ Categorização } & Análise ajustada & \multirow{2}{*}{ p-valor } \\
\hline & & ORa ajustada $\left({ }^{\prime} C_{95 \%}\right)^{b}$ & \\
\hline \multirow{2}{*}{ Sexo } & Feminino & - & - \\
\hline & Masculino & $1,39(1,05 ; 1,85)$ & 0,021 \\
\hline \multirow{4}{*}{ Faixa etária (em anos) } & $35-44$ & - & - \\
\hline & $45-54$ & $1,05(0,60 ; 1,81)$ & 0,856 \\
\hline & $55-64$ & $0,90(0,52 ; 1,54)$ & 0,700 \\
\hline & $\geq 65$ & $0,98(0,54 ; 1,76)$ & 0,954 \\
\hline \multirow{4}{*}{ Escolaridade } & Primeiro grau incompleto & $1,03(0,55 ; 1,96)$ & 0,905 \\
\hline & Primeiro grau completo & $1,38(0,74 ; 2,57)$ & 0,300 \\
\hline & Segundo grau completo & $0,95(0,62 ; 1,46)$ & 0,832 \\
\hline & Superior & - & - \\
\hline \multirow{5}{*}{$\begin{array}{l}\text { Renda per capita } \\
\text { (em salários mínimos) }\end{array}$} & $<4$ & $1,55(0,59 ; 4,06)$ & 0,370 \\
\hline & $4 a<8$ & $1,25(0,48 ; 3,23)$ & 0,633 \\
\hline & $8 a<12$ & $1,562(0,58 ; 4,18)$ & 0,375 \\
\hline & $12 \mathrm{a}<16$ & $3,02(0,59 ; 15,43)$ & 0,184 \\
\hline & $\geq 16$ & - & - \\
\hline \multirow{2}{*}{ Atividade religiosa } & Sim & - & - \\
\hline & Não & $0,87(0,64 ; 1,18)$ & 0,379 \\
\hline \multirow{4}{*}{ Raça/cor da pele } & Branca & - & - \\
\hline & Preta & $1,74(1,22 ; 2,48)$ & 0,002 \\
\hline & Parda & $1,57(1,14 ; 2,16)$ & 0,006 \\
\hline & Amarela/indígena & $1,65(0,91 ; 2,99)$ & 0,098 \\
\hline \multirow{3}{*}{ Categoria funcional } & Apoio & $1,67(0,94 ; 2,96)$ & 0,078 \\
\hline & Técnico & $1,63(1,02 ; 2,58)$ & 0,039 \\
\hline & Superior/docente & - & - \\
\hline \multirow{2}{*}{ Plano de saúde } & Sim & - & - \\
\hline & Não & $1,47(1,09 ; 1,96)$ & 0,010 \\
\hline \multirow{3}{*}{ Tipo de medicação } & Antidiabético oral & - & - \\
\hline & Insulina & $7,34(3,56 ; 15,15)$ & $<0,001$ \\
\hline & Ambos & $7,58(3,96 ; 14,52)$ & $<0,001$ \\
\hline \multirow{2}{*}{ Relação cintura-quadril (RCQ) } & Ideal & - & - \\
\hline & Alterada & $1,87(1,19 ; 2,93)$ & 0,007 \\
\hline \multirow{3}{*}{ Hábito de fumar } & Nunca fumou & - & - \\
\hline & Ex-fumante & $0,97(0,73 ; 1,28)$ & 0,817 \\
\hline & Fumante & $1,73(1,09 ; 2,74)$ & 0,019 \\
\hline \multirow{4}{*}{ Autoavaliação de saúde (AAS) } & Muito boa & - & - \\
\hline & Boa & $1,29(0,80 ; 2,09)$ & 0,290 \\
\hline & Regular & $1,53(0,93 ; 2,52)$ & 0,092 \\
\hline & Ruim/muito ruim & $2,37(1,17 ; 4,83)$ & 0,017 \\
\hline \multirow{4}{*}{ Calorias totais diárias da dieta } & $<2.000$ & - & - \\
\hline & $2.000<3.000$ & $0,89(0,64 ; 1,25)$ & 0,530 \\
\hline & $3.000<4.000$ & $1,17(0,78 ; 1,73)$ & 0,433 \\
\hline & $\geq 4.000$ & $1,53(0,95 ; 2,45)$ & 0,075 \\
\hline
\end{tabular}


Tabela 5 - Fatores associados ao controle glicêmico em indivíduos com diabetes mellitus participantes da linha de base do Estudo Longitudinal de Saúde do Adulto, Brasil, 2008-2010

\begin{tabular}{|c|c|c|c|}
\hline \multirow{2}{*}{ Variável } & \multirow{2}{*}{ Categorização } & Análise ajustada & \multirow{2}{*}{ p-valorc } \\
\hline & & ORa ajustada $\left(\mathrm{IC}_{95 \%}\right)^{\mathrm{b}}$ & \\
\hline \multirow{3}{*}{ Consumo de álcool } & Nunca usou & - & - \\
\hline & Ex-usuário & $0,92(0,60 ; 1,40)$ & 0,709 \\
\hline & Usuário & $0,97(0,64 ; 1,46)$ & 0,894 \\
\hline \multirow{2}{*}{ Adesão medicamentosa } & Baixa/média adesão & $1,20(0,92 ; 1,56)$ & 0,166 \\
\hline & Alta adesão & - & - \\
\hline
\end{tabular}

a) OR: odds ratio (razão de chances).

b) $\mathrm{IC}_{95 \%}$ : intervalo de confiança de $95 \%$.

c) A análise multivariada foi realizada por regressão logística binomial e incluiu todas as variáveis associadas ao resultado com $\mathrm{p}$-valor $<0,100$ na análise bivariada.

público, não apenas pela possibilidade de realizar o primeiro contato com um médico especialista, mas, sobretudo, pela frequência e facilidade com que os clientes de planos privados acessam o serviço de saúde, podendo ser este um fator determinante para um controle glicêmico adequado.

As variáveis relacionadas à doença, ao tratamento e à autopercepção da saúde aumentaram sobremaneira a chance de elevação da hemoglobina glicada. De acordo com o presente estudo, o uso de insulina também esteve relacionado com o aumento glicêmico, resultado compartilhado por outros pesquisadores. ${ }^{4}$ Esta relação, possivelmente, estaria relacionada às mudanças na rotina diária dos pacientes diabéticos, muitas vezes estressantes, provocadas pelo uso diário da insulina, necessidade de ajustes em seus hábitos diários, principalmente nos horários das refeições, além do armazenamento e transporte da medicação sob temperaturas controladas, uso de seringas para sua administração e descarte adequado de resíduos e de material perfurocortante. Estudo que investigou variáveis e sua potencial relação com o estresse em portadores de DM encontrou que a carga emocional e 0 estresse, - relacionados ao esquema terapêutico, ou nas relações interpessoais - estiveram associados ao uso de insulinoterapia, o que reforça ainda mais a carga emocional negativa proporcionada pelo uso $\mathrm{da}$ insulina no tratamento para o controle dessa doença. ${ }^{24}$

Ao lado do uso exclusivo da insulina, o tratamento com associação de insulina e antidiabéticos orais também contribuiu para um inadequado controle glicêmico de parte dos indivíduos com DM analisados. A necessidade de esquemas terapêuticos complexos, com a associação de insulinoterapia aos antidiabéticos orais, é uma prática necessária na rotina de diabéticos que não atingem as metas de controle glicêmico de outra forma. Estudo realizado para verificar o papel da polimedicação em indivíduos com DM tipo 2 ou hipertensos pertencentes a um grupo terapêutico, assistido por uma unidade da ESF em município do Sul do Brasil, verificou que os pacientes sob polimedicação apresentaram taxa de glicemia mais alta, comparados aos que utilizavam um único medicamento. ${ }^{25}$ Nesse sentido, a necessidade de esquemas complexos se apresenta como um grande desafio para o portador da doença, dada a necessidade de conhecimento especializado para seu manuseio, 0 que pode prejudicar o autocontrole metabólico.

Assim como neste estudo, pior autoavaliação de saúde também foi verificada entre diabéticos adultos coreanos com descontrole glicêmico. ${ }^{26}$ Para 0 diabético, a elevação da hemoglobina glicada pode representar pior condição de saúde, e, em função do conhecimento de seu processo metabólico, autoavaliação de sua saúde como ruim. Um dos fatores que também podem contribuir para a percepção negativa do estado de saúde pelo indivíduo com DM pode estar relacionado ao diagnóstico da doença em si, uma vez que sua descoberta implica, necessariamente, uma série de modificações no estilo de vida - e, sob a ótica do indivíduo diagnosticado, uma redução em sua qualidade de vida. ${ }^{27}$

Ser fumante contribuiu para pior controle glicêmico, tal qual um estudo realizado na Arábia Saudita observou: fumantes foram mais propensos a ter HbA1c mais elevada do que não fumantes. ${ }^{28}$ Além de estar associado ao descontrole metabólico, o tabagismo tem-se revelado um fator de risco para o desenvolvimento do DM. Outro estudo, realizado no Japão com trabalhadores de 12 companhias industriais, também identificou, mesmo 
após o ajuste de variáveis, incidência do DM tipo 2 entre os trabalhadores fumantes formais e informais, e risco de DM aumentado à medida que se observou aumento no consumo de cigarros entre os fumantes. ${ }^{29}$ De acordo com essas conclusões, o indivíduo com DM e fumante evidencia a não adaptação ao processo de doença, haja vista a manutenção de estilo de vida não condizente com sua situação clínica, podendo apresentar outras condições e comportamentos não favoráveis ao controle glicêmico.

A relação cintura-quadril (RCQ) alterada também esteve associada a controle glicêmico inadequado. Este achado difere de outros estudos, a não ser quando se refere apenas à incidência de casos de DM tipo $2 .{ }^{30} \mathrm{~A} \mathrm{RCQ}$ alterada pode ser reflexo da alteração dos parâmetros nutricionais da maioria dos participantes, especialmente dos que apresentaram um controle glicêmico inadequado. Apresentaram sobrepeso e obesidade, somados, 82,8\%; e destes, quase $40 \%$ tinham obesidade abdominal e mais de $90 \%$ valores alterados para circunferência da cintura. Outrossim, 56,2\% consumiam bebida alcoólica regularmente, 96,6\% eram inativos fisicamente, e mais de um terço referiu dieta com mais de 3.000 calorias/dia. Essas alterações também foram observadas metabolicamente: 54,2\% apresentaram valores de HbAlc $>6,5 \%$, evidenciando um descontrole metabólico.

Uma limitação desta pesquisa se encontra no desenho transversal, que não permite investigar a relação de temporalidade com grande parte das variáveis. Todavia, trata-se de um estudo exploratório, capaz de levantar

\section{Referências}

1. Sociedade Brasileira de Diabetes (BR). Diretrizes da Sociedade Brasileira de Diabetes 2017-2018 [Internet]. In: Oliveira JEP, Montenegro Júnior RM, Vencio S (organizadores). São Paulo: Editora Clannad; 2017 [citado 2020 maio 19]. 383 p. Disponível em: https://www.diabetes.org.br/profissionais/ images/2017/diretrizes/diretrizes-sbd-2017-2018.pdf

2. International Diabetes Federation. IDF Diabetes atlas [Internet]. $7^{\text {th }}$ ed. [S.I.]: International Diabetes Federation; 2015 ]cited 2020 May 19]. 142 p. Available from: https://www.idf.org/e-library/epidemiologyresearch/diabetes-atlas/13-diabetes-atlas-seventhedition.html hipóteses a serem respondidas com estudos de coorte e, ao mesmo tempo, propiciar informações para os serviços de saúde, subsidiando a promoção da saúde dos portadores de diabetes mellitus.

Os resultados apresentados reforçam o contexto multicausal e seu poder para atuar como barreira ou facilitador do controle glicêmico, considerando-se a complexidade da interação dos determinantes sociais de saúde nesse processo, ao passo que enfoca várias dimensões, como, por exemplo, a qualidade, quantidade e frequência da dieta, tipo, regularidade e tempo de atividade física, a ausência de obesidade, controle do consumo de álcool, além da tomada correta da medicação, supostamente prescrita de forma adequada.

Conclui-se pela necessidade da implementação de linhas de cuidado do diabético mediante o fortalecimento do modelo de atenção às condições crônicas, inserindo-0 na rede de atenção ao doente crônico.

\section{Contribuição dos autores}

Moraes HAB participou da concepção, planejamento, interpretação dos dados e redação do manuscrito. Mengue SS e Molina MCB participaram da revisão crítica do conteúdo do manuscrito. Cade NV participou da concepção, planejamento, interpretação dos dados e redação da versão final do manuscrito. Todos os autores aprovaram a versão final do manuscrito e são responsáveis por todos os aspectos do trabalho, incluindo a garantia de sua precisão e integridade.

3. Iser BPM, Stopa SR, Chueiri OS, Szwarcwald CL Malta DC, Monteiro HOC, et al . Prevalência de diabetes autorreferido no Brasil: resultados da Pesquisa Nacional de Saúde 2013. Epidemiol Serv Saúde [Internet]. 2015 abr-jun [citado 2020 fev 5]; 24(2):305-14. Disponível em: https://doi.org/10.5123/ S1679-49742015000200013

4. Panarotto D, Teles AR, Schumacher MV. Fatores associados ao controle glicêmico em pacientes com diabetes tipo 2. Rev Assoc Med Bras [Internet]. 2008 [citado 2020 maio 19];54(4):314-21. Disponível em: https://www.scielo.br/pdf/ramb/v54n4/15.pdf 
5. Morais GFC, Soares MJG0, Costa MML, Santos IBC. 0 diabético diante do tratamento, fatores de risco e complicações crônicas. Rev Enferm UERJ [Internet] 2009 abr-jun [citado 2020 maio 19];17(2):240-5. Disponível em: https://www.facenf.uerj.brv17n2/ v17n2a18.pdf

6. Aquino EML, Araujo MJ, Almeida MCC, Conceição P, Andrade CR, Cade NV et al. Recrutamento de participantes no estudo longitudinal de saúde do adulto. Rev Saúde Pública [Internet]. 2013 jun [citado 2020 maio 19];47(Suppl 2):10-8. Disponível em: https://doi.org/10.1590/S0034-8910.2013047003953

7. Bensenor IM, Griep RH, Pinto KA, Faria KP, FelisbinoMendes M, Caetano EI, et al. Rotinas de organização de exames e entrevistas no centro de investigação ELSA-Brasil. Rev Saúde Pública [Internet]. 2013 jun [citado 2020 maio 19];47(2):37-47. Disponível em: https://doi.org/10.1590/S0034-8910.2013047003780

8. American Diabetes Association. Classification and diagnosis of diabetes. Diabetes Care [Internet]. 2016 Jan [cited 2020 May 19];39(suppl 1):S4-5. Available from: https://doi.org/10.2337/dc16-S003

9. Molina MCB, Benseñor IM, Cardoso LO, VelasquezMelendez G, Drehmer M, Pereira TSS, et al. Reprodutibilidade e validade relativa do Questionário de Frequência Alimentar do ELSA-Brasil. Cad Saúde Pública [Internet]. 2013 fev [citado 2020 maio 19];29(2):379-89. Disponível em: https://doi. org/10.1590/S0102-311X2013000200024

10. Morisky DE, Ang A, Krousel-Wood M, Ward HJ. Predictive validity of a medication adherence measure in an outpatient setting. J Clin Hypertens (Greenwich) [Internet]. 2008 May [cited 2020 May 19];10(5):34854. Available from: https://doi.org/10.1111/j.17517176.2008.07572.x.

11. Remondi FA, Cabrera MAS, Souza RKT. Não adesão ao tratamento medicamentoso contínuo: prevalência e determinantes em adultos de 40 anos e mais. Cad Saúde Pública [Internet]. 2014 jan [citado 2020 maio 19];30(1):126-36. Disponível em: https://doi. org/10.1590/0102-311X00092613

12. Ben AJ, Neumann CR, Mengue SS. Teste de MoriskyGreen e Brief Medication Questionnaire para avaliar adesão a medicamentos. Rev Saúde Pública [Internet] 2012 fev [citado 2020 maio 19]; 46(2):279-89. Disponível em: https://doi.org/10.1590/S003489102012005000013
13. International Diabetes Federation - IDF. The IDF consensus worldwide definition of the metabolic syndrome [Internet]. Brussels: International Diabetes Federation; 2006 [cited 2020 May 19]. 23 p. Available from: https://www.idf.org/e-library/consensusstatements/60-idfconsensus-worldwide-definitionofthe-metabolic-syndrome.html

14. Associação Brasileira para Estudo da Obesidade e da Síndrome Metabólica (BR) - ABESO. Diretrizes brasileiras de obesidade 2009/2010 [Internet]. 3. ed. Itapevi: AC Farmacêutica; 2009 [citado 2020 maio 19]. 83 p. Disponível em: http://www.saude.df.gov.br/ wp-conteudo/uploads/2018/08/2009_DIRETRIZES BRASILEIRAS_DE_OBESIDADE.pdf

15. Hallal PC, Matsudo SM, Matsudo VKR, Araújo TL, Andrade DR, Bertoldi AD. Physical activity in adults from two Brazilian areas: similarities and differences. Cad Saúde Pública [Internet]. 2005 Mar-Apr [cited 2020 May 19];21(2):573-80. Available from: http:// dx.doi.org/10.1590/S0102-311X2005000200024

16. Ministério da Saúde (BR). Secretaria de Atenção à Saúde. Política nacional de atenção integral à saúde do homem: princípios e diretrizes [Internet]. Brasília: Ministério da Saúde; 2008 [citado 2020 maio 19]. 40 p. Disponível em: https://bvsms.saude.gov.br/bvs/ publicacoes/politica_nacional_atencao_homem.pdf

17. Gomes R, Nascimento EF, Araújo FC. Por que os homens buscam menos os serviços de saúde do que as mulheres? As explicações de homens com baixa escolaridade e homens com ensino superior. Cad Saúde Pública [Internet]. 2007 mar [citado 2020 maio 19];23(3):565-74. Disponível em: http://dx.doi. org/10.1590/S0102-311X2007000300015

18. Chiu M, Austin PC, Manuel DG, Tu JV. Comparison of cardiovascular risk profiles among ethnic groups using population health surveys between 1996 and 2007. CMAJ [Internet]. 2010 May [cited 2020 May 19];182(8):301-10. Available from: https://doi. org/10.1503/cmaj.091676

19. Lafata JE, Karter AJ, O'Connor PJ, Morris H, Schmittdiel JA, Ratliff S, et al. Medication adherence does not explain black-white differences in cardiometabolic risk factor control among insured patients with diabetes. J Gen Intern Med [Internet]. 2016 Feb [cited 2020 May 19];31(2):188-95. Available from: https://doi.org/10.1007/s11606-0153486-0 
20. Ministério da Saúde (BR). Secretaria de Vigilância em Saúde. Departamento de Vigilância de Doenças e Agravos não Transmissíveis e Promoção da Saúde. Vigitel Brasil 2014: vigilância de fatores de risco e proteção para doenças crônicas por inquérito telefônico [Internet]. Brasília: Ministério da Saúde; 2015 [citado 2020 maio 19]. 152 p. Disponível em: https://bvsms.saude.gov.br/bvs/publicacoes/vigitel_ brasil_2014.pdf

21. Schmidt MI, Hoffmann JF, Diniz MFS, Lotufo PA, Griep RH, Bensenor IM, et al. High prevalence of diabetes and intermediate hyperglycemia: the brazilian longitudinal study of adult health (ELSA-Brasil) [Internet]. Diabetol Metab [Internet]. 2014 Nov [cited 2020 May 19];6(123). Available from: https://doi. org/10.1186/1758-5996-6-123

22. Carvalho AI. Determinantes sociais, econômicos e ambientais da saúde. In: Fundação Oswaldo Cruz (BR). A saúde no Brasil em 2030 - prospecção estratégica do sistema de saúde brasileiro: população e perfil sanitário [Internet]. Rio de Janeiro: Fiocruz/ Ipea/Ministério da Saúde; 2013 [citado 2020 maio 19]. p. 19-38. Disponível em: https://saudeamanha. fiocruz.br/wp-content/uploads/2016/07/11.pdf

23. Silva SS, Mambrini JVM, Turci MA, Macinko J, LimaCosta MF. Uso de serviços de saúde por diabéticos cobertos por plano privado em comparação aos usuários do Sistema Único de Saúde no Município de Belo Horizonte, Minas Gerais, Brasil. Cad Saúde Pública [Internet]. 2016 out [citado 2020 maio 19];32(10):e00014615. Disponível em: https://doi. org/10.1590/0102-311X00014615

24. Zanchetta FC, Trevisan DD, Apolinario PP, Silva JB, Lima MH. Variáveis clínicas e sociodemográficas associadas com o estresse relacionado ao diabetes em pacientes com diabetes mellitus tipo 2. Einstein [Internet]. 2016 jul-set [citado $2017 \mathrm{abr}$ 11];14(3):346-51. Disponível em: https://doi. org/10.1590/\$1679-45082016A03709
25. Ames KS, Bassani PH, Motter N, Roratto B, Hammes JLN, Quadro MN, et al. Avaliação de hipertensos e diabéticos usuários de polimedicação em Santo Ângelo/RS. Rev Sau Int [Internet]. 2016 [citado 2020 maio 19];9(17):58-65. Disponível em: http://local. cnecsan.edu.br/revista/index.php/saude/index

26. Lee HW, Song M, Yang JJ, Kang D. Determinants of poor self-rated health in korean adults with diabetes. J Prev Med Public Health [Internet]. 2015 Nov [citado 2020 May 19];48(6):287-300. Available from: https:// dx.doi.org/10.3961\%2Fjpmph.15.048

27. Feng X, Astell-Burt T. Impact of a type 2 diabetes diagnosis on mental health, quality of life, and social contacts: a longitudinal study. BMJ Open Diabetes Res Care [Internet]. 2017 [cited 2020 May 19];5:e000198. Available from: http://dx.doi.org/10.1136/ bmjdrc-2016-000198

28. Badedi M, Solan Y, Hussain D, Sabai A, Mahfouz M, Alamodi S, et al. Factors associated with long-term control of type 2 diabetes mellitus. J Diabetes Res [Internet]. 2016 Dec [cited 2020 May 19];2016:2109542. Available from: https://doi. org/10.1155/2016/2109542

29. Akter S, Okazaki H, Kuwahara K, Miyamoto T, Murakami T, Shimizu C, et al. Smoking, smoking cessation, and the risk of type 2 diabetes among japanese adults: japan epidemiology collaboration on occupational health study. PLoS One [Internet]. 2015 Jul [cited 2020 May 19];10(7):e0132166. Available from: https://doi.org/10.1371/journal.pone.0132166

30. Diabetes Prevention Program Research Group, Knowler WC, Fowler SE, Hamman RF, Brenneman AT, et al.10-year follow-up of diabetes incidence and weight loss in the Diabetes Prevention Program Outcomes Study. Lancet [Internet]. 2009 Nov [cited 2020 May 19];374(9702):1677-86. Available from: https://doi.org/10.1016/s0140-6736(09)61457-4 


\begin{abstract}
Objective: to investigate factors associated with glycemic control in individuals with diabetes mellitus (DM). Methods: this was a cross-sectional study, with participants in the Longitudinal Study of Adult Health with self-reported DM; binomial logistic regression was used. Results: 1,242 individuals were included; $54.2 \%$ had glycated hemoglobin $\geq 6.5 \%$, showing inadequate glycemic control; factors associated with inadequate glycemic control were male sex $(O R=1.39-95 \%$ CI 1.05;1.85), black skin color $(O R=1.74-95 \%$ CI 1.22;2.48) or brown skin color ( $O R=1,57-95 \%$ CI 1.14;2.16), average occupation level $(O R=1.63-95 \% C I$ 1.02;2.58), not baving bealth insurance (OR=1.47-95\%CI 1.09;1.96), use of insulin $(O R=7.34-95 \% C I 3.56 ; 15.15)$, increased waist-to-hip ratio $(O R=1.87-95 \% C I 1.19 ; 2.93)$, smoking $(O R=1.73$ -95\%CI 1.09;2.74), and poor or very poor self-rated health $(O R=2.37-95 \% C I$ 1.17;4.83). Conclusion: the results reinforce the multicausal context in glycemic control, which was associated with sociodemographic factors, lifestyles and health conditions.
\end{abstract}

Keywords: Diabetes Mellitus; Glycated Hemoglobin A; Diabetes Complications; Hyperglycemia.

\section{Resumen}

Objetivo: investigar los factores asociados con el control glucémico en personas con diabetes mellitus (DM). Métodos: estudio seccional, con participantes en el Estudio Longitudinal de Salud del Adulto con DM autoinformada; se utilizó regresión logística binomial. Resultados: se incluyeron 1.242 individuos; el 54,2\% tenía hemoglobina glicada $\geq 6,5 \%$, mostrando un control glucémico inadecuado; los factores asociados con un control glucémico inadecuado fueron género masculino $\left(O R=1,39-I C_{95 \%} 1,05 ; 1,85\right)$, raza/color de piel negra $\left(O R=1,74-I C_{95 \%} 1,22 ; 2,48\right)$ o parda $\left(O R=1,57-I C_{95 \%} 1,14 ; 2,16\right)$, nivel medio de ocupación $\left(O R=1,63-I C_{95 \%} 1,02 ; 2,58\right)$, sin seguro de salud $\left(O R=1,47-I C_{95 \%} 1,09 ; 1,96\right)$, uso de insulina $(O R=7,34$ $\left.-I C_{9500} 3,56 ; 15,15\right)$, aumento de la relación cintura-cadera $\left(O R=1,87-I C_{95_{\circ}^{\circ}} 1,19 ; 2,93\right)$, tabaquismo (OR=1,73$\left.I C_{95 \%} 1,09 ; 2,74\right)$ y autoevaluación de mala salud o muy mala $\left(O R=2,37-I C_{95 \%} 1,17 ; 4,83\right)$. Conclusión: los resultados refuerzan el contexto multicausal en el control glucémico, que se asoció con factores sociodemográficos, estilos de vida y condiciones de salud.

Palabras clave: Diabetes Mellitus; Hemoglobina A Glucada; Complicaciones de la Diabetes; Hiperglucemia.

Recebido em 25/01/2019

Aprovado em 20/04/2020 